\title{
Souris transgéniques et lignées cellulaires: outils pour une approche moléculaire de la différenciation germinale
}

La somme de connaissances acquises sur la structure et la physiologie du testicule permet d'aborder l'étude des signaux et des facteurs impliqués dans les régulations transcriptionnelles et post-transcriptionnelles intervenant au cours de la spermatogenèse. Une liste de gènes dont l'expression est spécifique de l'un des stades de différenciation a pu être établie. Mais parmi ceux-ci, peu de facteurs régulateurs ont été clairement identifiés. Les outils nécessaires commencent en fait seulement à être disponibles. C'est maintenant la construction de souris transgéniques et les techniques d'inactivation par recombinaison homologue qui vont permettre de cerner au plus près la fonction d'un gène. Ces techniques restent, cependant, assez lourdes à mettre en œuvre et présentent des limitations intrinsèques. Elles doivent être associées à des analyses menées en culture de cellules. Là aussi, les modèles expérimentaux se sont affinés, fondés sur l'établissement de lignées germinales et de lignées somatiques testiculaires.

\section{Expression de gènes régulateurs au} cours de la spermatogenèse

Plusieurs gènes à fonction régulatrice connue ou supposée sont exprimés à des stades définis de la spermatogenèse $[1,2]$ (Tableau I). Ils ont été trouvés, soit en criblant des banques d'expression avec des sondes correspondant à des séquences consensus de fixation à l'ADN (clonage du gène Zfp35 [3]), soit parce qu'ils étaient déjà connus dans d'autres tissus (ainsi la protéine p53, exprimée au stade pachytène [4]). Leurs rôles restent néanmoins à déterminer. Pour ceux dont l'expression n'est pas restreinte aux cellules germinales, l'analyse peut s'appuyer sur les connaissances acquises dans d'autres tissus. Une fonction au cours de la spermatogenèse a été proposée pour les gènes sry [5], sox 5 [6], zfp35 [3], cftn51 [7] $A Z F$ [8] ou sperm1 [9]. L'importance de sry au cours du développement du testicule est maintenant démontrée [10], mais son rôle éventuel au cours de la spermatogenèse reste obscur : les transcrits sont trouvés sous une forme circulaire, et aucune protéine n'a pu être détectée. Un candidat intéressant est le produit de sperm 1, qui possède un domaine $\mathrm{POU}^{*}$ retrouvé dans plusieurs protéines impliquées dans le développement du système nerveux. sperm1, transcrit aux stades pachytène et diplotène, pourrait participer à la régulation de l'entrée en méiose $\left(m / s n^{\circ} 3\right.$, vol. $\left.10, p .344\right)$.

\section{La souris transgénique, un outil} nécessaire

Les méthodes actuelles d'accès au gène impliquent l'utilisation, soit de cellules en culture, soit d'animaux transgéniques. Les techniques de transgénèse, lourdes et coûteuses, présentent l'avantage déterminant d'éviter tous les aspects artificiels, et obligatoires, de la culture de cellules. La voie aujourd'hui la plus prometteuse est l'analyse des conséquences phénotypiques de l'inactivation d'un gène par recombinaison homologue.

\footnotetext{
* POU : domaine de fixation à l'ADN, toujours trouvé associè à une homéobồte.
}

Cependant, si une fonction peut être ainsi identifiée sans ambiguïté, il est apparu que, dans beaucoup de cas, la fonction de la protéine absente est relayée par d'autres, masquant ainsi son véritable rôle. On ne pourra conclure d'un résultat négatif. Ainsi, l'inactivation de mos, exprimé pendant la méiose [11], et celle de p53 [12] ont montré qu'aucun de ces deux gènes n'est nécessaire à la spermatogenèse, mais n'ont pas exclu qu'ils y aient une fonction. De même, l'inactivation du gène $C R E B$ (cyclic AMP responsive element binding protein) impliqué dans le contrôle de l'expression génique au cours de la spermatogenèse (voir l'article de P. Sassone-Corsi, p. 616 de ce numéro) ne modifie ni le développement ni la reproduction de la souris, car les autres membres de cette famille de facteurs de transcription suppléent cette inactivation [13]. Parmi les techniques les plus récentes de transgénèse ciblée, la recombinaison dirigée aux sites loxp, telle qu'elle a été utilisée pour la polymérase $\beta$ dans les lymphocytes $\mathrm{T}$ [14], permet l'inactivation d'un gène, dans le tissu et au stade de développement choisis. Les applications à la différenciation germinale en sont attendues avec intérêt.

La construction d'animaux transgéniques qui expriment un gène rapporteur sous contrôle des séquences régulatrices des gènes étudiés a, par ailleurs, permis d'identifier, dans un premier temps, les régions impliquées en cis dans les régulations, puis les facteurs qui reconnaissent cellesci. Cela a été réalisé pour les gènes prm1 [15], pgk2 [16], tcp-10 [17], Hox-a.4 [18], zfy1 [19]. Nous détaille- 
rons à titre d'exemple l'étude de pgk2 et prm1.

La phosphoglycérate kinase 2 (gène $p g k 2$ ) est une isoenzyme spécifique des cellules germinales. L'ARN messager est transcrit pendant la méiose, puis accumulé jusqu'au stade spermatide ronde avant d'être traduit. Dans des expériences de transgénèse, une région de $0,5 \mathrm{~kb}$ en amont du site de démarrage de la transcription a été définie comme nécessaire et suffisante pour que l'expression d'un transgène soit parfaitement concomitante de celle du gène [16]. Elle comporte une séquence de douze paires de bases, commune au promoteur du gène $z f p 35$ qui est exprimé au même stade de la spermatogenèse. Une protéine fixant cette séquence a été identifiée par criblage d'une banque d'expression d'ADNc de testicule. Cette protéine, appelée tsHMG, est présente aux stades où l'expression des gènes pgk2 et zfp35 s'éteint [20]. Elle possède un domaine HMG (high mobility group), caractéristique d'un facteur de transcription et retrouvé dans les protéines SRY et Sox5. Le rôle de ces deux derniers facteurs reste inconnu, mais la protéine tsHMG pourrait agir comme un inhibiteur transcriptionnel, à un stade où la transcription de plusieurs gènes est réprimée dans les cellules germinales.

Le gène prm1 code pour la protamine 1 , une des protéines qui remplacent les histones après la méiose. Il est transcrit au stade spermatide ronde, mais les messagers ne sont traduits qu'une semaine plus tard, au stade spermatide allongée. Dans des souris transgéniques, le déclenchement de la transcription dépend d'une séquence en amont du gène [15] alors qu'une partie de la région 3' non codante du messager paraît responsable du délai de traduction [21]. En amont du gène, le site de fixation d'un facteur spécifique des cellules germinales post-méiotiques a été identifié. Il contient une séquence proche d'un élément de réponse à l'AMPc (séquence CRE-like) qui fixe un facteur appelé Tet-1 [22]. Des mutations dans cette séquence réduisent l'activité du promoteur [23].

\begin{tabular}{|c|c|c|c|}
\hline & $\begin{array}{r}\text { QUELQUES PROT } \\
\text { UNE FONC } \\
\text { COURS DE LA SPE }\end{array}$ & $\begin{array}{l}\text { Tableau I } \\
\text { EINES POUR LESQUELLES } \\
\text { ION RÉGULATRICE } \\
\text { MATOGENÉSE EST PROPO }\end{array}$ & SÉE \\
\hline Protéines & Stade & Rôle proposé & Réf. \\
\hline $\begin{array}{l}\text { Protéines à } \\
\text { tsHMG } \\
\text { SRY, Sox } 5\end{array}$ & $\begin{array}{l}\text { "boîte HMG " } \\
\text { spermatide allongé } \\
\text { spermatide ronde }\end{array}$ & $\begin{array}{l}\text { inhibiteur transcriptionnel } \\
?\end{array}$ & $\begin{array}{l}{[20]} \\
{[5][6]}\end{array}$ \\
\hline $\begin{array}{l}\text { Protéines fi } \\
\text { CREM } \\
\text { CREM } \tau \\
\text { Tet-1 }\end{array}$ & $\begin{array}{l}\text { sant les motifs CRE } \\
\text { préméiotique } \\
\text { postméiotique } \\
\text { postméiotique }\end{array}$ & $\begin{array}{l}\text { CRE-LIKE } \\
\text { inhibiteur transcriptionnel } \\
\text { activateur transcriptionnel } \\
\text { activateur transcriptionnel }\end{array}$ & $\begin{array}{l}\text { P. Sassone- } \\
\text { Corsi, (p. } 616 \\
\text { de ce numé- } \\
\text { ro) [22] }\end{array}$ \\
\hline $\begin{array}{l}\text { Protéines à } \\
\text { Zpf-35' } \\
\text { Ctfn51 }\end{array}$ & $\begin{array}{l}\text { "doigts de zinc" } \\
\text { pachytène } \\
\text { pachytène à } \\
\text { spermatide ronde }\end{array}$ & contrôle de la méiose & $\begin{array}{l}{[3]} \\
{[7]}\end{array}$ \\
\hline $\begin{array}{l}\text { Protéines à } \\
\text { Sperm } 1\end{array}$ & $\begin{array}{l}\text { "domaine POU " } \\
\text { spermatocyte } 1\end{array}$ & facteur de différenciation & [9] \\
\hline $\begin{array}{l}\text { Protéines s } \\
\text { AZF }\end{array}$ & $\begin{array}{l}\text { liant à I'ARN } \\
\text { testicule adulte }\end{array}$ & $\begin{array}{l}\text { épissage des ARNm, } \\
\text { contrôle traductionnel }\end{array}$ & [8] \\
\hline $\begin{array}{l}\text { Protéine kin } \\
\text { MPF } \\
\text { p53 } \\
\text { c-Mos } \\
\text { c-Abl }\end{array}$ & $\begin{array}{l}\text { ase et proto-oncogè } \\
\text { pachytène } \\
\text { mid-pachytène } \\
\text { fin méiose } \\
\text { spermatide ronde }\end{array}$ & $\begin{array}{l}\text { contrôle de la méiose? } \\
\text { intégrité du génome? } \\
\text { arrêt des divisions? } \\
\text { morphologie des cellules } \\
\text { après la méiose? }\end{array}$ & $\begin{array}{l}{[36]} \\
{[12]} \\
{[11]} \\
{[37]}\end{array}$ \\
\hline
\end{tabular}

teurs des gènes $A C E$ [24] et pim1 [25], exprimés au même stade de la spermatogenèse. Il reste à déterminer le rôle du facteur Tet-1, et de ses interactions avec les séquences CRElike.

Moins de résultats ont été obtenus en ce qui concerne les régulations posttranscriptionnelles de l'expression génique dans les cellules germinales. Les études les plus poussées ont été réalisées sur le gène de la protamine 2 , exprimé aux mêmes stades que la protamine 1, et dont l'ARNm est accumulé une semaine avant d'être traduit. Au moins une protéine se lierait, une fois phosphorylée, à une séquence de la partie 3' non codante de l'ARNm, et inhiberait sa traduction [26].
Modèles expérimentaux en culture de cellules

Comme la majorité des cellules différenciées, les cellules germinales ne peuvent être maintenues à long terme en culture (voir [27] pour revue). $\mathrm{Si}$ les régulations endocrines d'origine extérieure au tube séminifère (hypophyse, cellules de Leydig, cellules péritubulaires) peuvent être assurées par des apports adéquats dans le milieu de culture, il est clair que la différenciation des cellules germinales dépend étroitement de leur environnement dans l'épithélium séminifère, particulièrement de leur association aux cellules de Sertoli. On pourra, d'une part, chercher à établir des lignées de cellules germi- 
Tableau ॥

LIGNÉES MURINES ÉTABLIES UTILISABLES POUR LES EXPÉRIENCES DE SPERMATOGENĖSE IN VITRO

\begin{tabular}{|c|c|c|c|}
\hline Lignée & Immortalisation par & Caractéristiques & Réf. \\
\hline GC-1spg & SVLT & $\begin{array}{l}\text { - cellules germinales entre spermatogonie B } \\
\text { et spermatocyte préleptotène } \\
\text { - étude du promoteur du gène ldhx }\end{array}$ & [29] \\
\hline $\begin{array}{l}\text { GC-2spd(ts) } \\
\text { GC-3spc(ts) }\end{array}$ & SVLT+p53ts & $\begin{array}{l}\text { - cellules germinales préméiotiques } \\
\text { - passage de la méiose in vitro dans les conditions permissives pour p53 }\end{array}$ & [31] \\
\hline $15 P-1$ & PyLT & $\begin{array}{l}\text { - cellules de Sertoli } \\
\text { - passage de la méiose in vitro par des cellules germinales primaires } \\
\text { en coculture }\end{array}$ & [34] \\
\hline
\end{tabular}

nales modifiées pour être capables d'une maturation autonome. Toute lignée qui reproduirait in vitro une étape, même limitée, de la différenciation germinale serait d'un grand intérêt. L'alternative sera de reconstituer dans des cultures mixtes ("COcultures») l'association étroite que l'on connaît in vivo entre les cellules germinales et les cellules de Sertoli. Les contacts directs et les échanges de signaux entre les deux types cellulaires sont l'un des aspects fondamentaux de la spermatogenèse; ils devraient être, au moins en partie, conservés.

L'immortalisation de cellules différenciées a été réalisée pour d'autres types cellulaires par l'expression d'oncogènes immortalisants (voir [28] pour revue), notamment ceux qui codent pour les protéines $\mathrm{T}$ des virus SV40 (SVLT) et polyome (PyLT). Cependant, cette étape d'immortalisation engage la cellule dans un processus de progression tumorale, dont les stades avancés risquent d'être peu compatibles avec l'expression de caractères différenciés. Une solution est l'utilisation de mutants conditionnels de l'oncogène ou d'un anti-oncogène. Une autre solution est d'utiliser la protéine PyLT, dont le pouvoir oncogène est essentiellement limité à l'immortalisation. Trois lignées germinales ont récemment été isolées après transformation par SV40 (protéine SVLT). Les cellules de la lignée GC-1spg [29] expriment deux marqueurs de stades préméio$m / s n^{\circ} 4$, vol. II, avril 95 tiques, la sous-unité C4 de la lactate déshydrogénase (gène $l d h x$ ) et le cytochrome Ct. Elles sont arrêtées à ce stade de différenciation. La transfection dans ces cellules de gènes rapporteurs sous contrôle de régions génomiques en amont du gène $l d h x$ a permis de définir le promoteur minimal de ce gène [30]. Dans les lignées GC-2spd(ts) et GC-3spc(ts) [31], le pouvoir transformant de SVLT a été limité par la co-expression d'une version sensible à la température du suppresseur de tumeur p53. A température restrictive, les cellules se divisent sans exprimer de caractères différenciés. Aux températures permissives, p53 bloque l'action transformante de la protéine SVLT et les cellules expriment alors les marqueurs de différenciation cités précédemment. Un certain nombre d'entre elles passent la méiose, produisant une fraction de cellules haploïdes, repérées par leur contenu en $\mathrm{ADN}$, et dans lesquelles un acrosome commence à se former. Il sera intéressant de dresser une liste précise des marqueurs moléculaires exprimés par ces cellules qui, si leurs propriétés et leur caryotype restent stables au cours des passages, constitueront un instrument important d'étude des régulations de l'expression génique avant et pendant la méiose.

La coculture n'a pu être réalisée de manière efficace en utilisant des cultures primaires de cellules somatiques testiculaires. Les lignées sertoliennes établies à partir de tumeurs ou après transformation par SV40 ne gardent que très partiellement leurs caractères différenciés [29, 32]. D'autres lignées ont été établies à partir de souris transgéniques, exprimant dans l'épithélium séminifère la protéine PyLT [33]. Elles conservent certains des caractères structuraux et moléculaires des cellules de Sertoli. Une d'entre elles au moins, 15P-1, permet la différenciation "transméiotique" de cellules germinales primaires en coculture. Des cellules germinales, prélevées avant le déclenchement de la première méiose chez un mâle immature, expriment successivement dans ces cultures mixtes des marqueurs précoces de méiose (cycline A, D. Wolgemuth, communication personnelle), puis le gène prm1 (protamine 1), à expression strictement post-méiotique, et des noyaux haploïdes s'accumulent $(\mathrm{m} / \mathrm{s}$ $n^{\circ} 4$, vol. 10, p. 466) [34]. L'expression de différents gènes peut être suivie dans ces cocultures. Il devrait être possible de définir les séquences régulatrices de leur expression par transfection d'un gène rapporteur sous contrôle de ces séquences. Cependant, les résultats préliminaires de différents laboratoires tendent à montrer que les cellules germinales sont difficilement transfectables. Le système de coculture se prête particulièrement bien à l'analyse des signaux transmis entre la cellule de Sertoli et la cellule germinale. Des expériences en cours (S. Vincent et M. Rassoulzadegan, non publié) ont 
permis d'identifier une série de cytokines sécrétées par la cellule 15P-1 (KL, CSF-1, NGF, FGF-1, notamment). L'utilisation d'anticorps monoclonaux contre ces facteurs et leurs récepteurs, d'oligonucléotides antisens, d'inhibiteurs des voies de transduction du signal en aval des récepteurs devrait permettre d'identifier certains des éléments impliqués dans les "conversations" entre cellules de Sertoli et cellules germinales.

\section{Conclusion}

De plus en plus de gènes sont connus pour être exprimés aux différents stades de la spermatogenèse. Cependant, aucune réponse n'a pour l'instant été apportée à la question de l'existence possible de "gènes maîtres ", qui contrôleraient le processus de différenciation, comme il en a été trouvé pour d'autres voies de différenciation [35]. Une partie des difficultés méthodologiques sont maintenant levées, puisque nous pouvons associer les méthodes de transgénèse, relativement lourdes, mais indispensables, avec l'utilisation de lignées de cellules germinales et de cocultures de cellules germinales et somatiques. La voie paraît ainsi ouverte à une analyse moléculaire fine des régulations

\section{Julien Sage, François Cuzin}

Inserm U.273, Faculté des Sciences, 06108 Nice Cedex, France.

\section{RÉFÉRENCES}

1. Barouki R. Expression des gènes au cours de la spermatogenèse. médecine/sciences $1992 ; 8: 532-40$.

2. Wolgemuth DJ, Watrin F. List of cloned mouse genes with unique expression patterns during spermatogenesis. Mammalian Genome 1991; 1 : 283-8.

3. Cunliffe V, Koopman P, McLaren A, Trowsdale J. A mouse zinc finger gene which is transiently expressed during spermatogenesis. EMBO J $1990 ; 9$ : 197-205.

4. Schwartz D, Goldfinger N, Rotter V. Expression of p53 in spermatogenesis is confined to the tetraploid pachytene primary spermatocytes. Oncogene $1993 ; 8$ : 1487-94.

5. Capel B, Swain A, Nicolis S, Hacker A, Walter M, Koopman P, Goodfellow P, Lovell-Badge $\mathrm{R}$. Circular transcripts of the testis-determining gene sry in adult mouse testis. Cell $1993 ; 73: 1019-30$.

6. Denny P, Swift S, Connor F, Ashworth A. An $S R Y$-related gene expressed during spermatogenesis in the mouse encodes a sequence-specific DNA-binding protein. EMBO 1992; 11 : 3705-12.

7. Noce T, Fujiwara Y, Sezaki M, Fujimoto $\mathrm{H}$, Higashinakagawa T. Expression of a mouse zinc finger protein gene in both spermatocytes and oocytes during meiosis. Development 1992 ; 153 : 356-67.

8. Ma K, Inglis JD, Sharkey A, Bickmore WA, Hill RE, Prosser EJ, Speed RM, Thomson EJ, Jobling $\mathbf{M}$, Taylor $\mathbf{K}$, Wolfe J, Cooke HJ, Hargreave TB, Chandley AC. A Y chromosome gene family with RNA-binding protein homology: candidates for azoospermia factor AZF controlling human spermatogenesis. Cell 1993 ; 75 : 1287-95.

9. Andersen B, Pearse RV, Schlegel PN, Cichon Z, Schonemann MD, Bardin CW, Rosenfeld MG. Sperm 1: a POU domain gene transiently expressed immediately before meiosis I in the male germ cell. Proc Natl Acad Sci USA 1993 ; 90 : 11084-8.

10. Koopman P, Gubbay J, Vivian N, Goodfellow P, Lovell-Badge R. Male development of chromosomally female mice transgenic for Sry. Nature 1991 ; 351 : 117-21.

11. Colledge WH, Carlton MBL, Udy GB, Evans M]. Disruption of c-mos causes parthenogenetic development of unfertilized mouse eggs. Nature $1994 ; 370$ : 65-8.

12. Donehower LA, Harvey M, Slagle BT, McArthur MJ, Montgomery MA, Butel JS, Bradley A. Mice deficient for p53 are developmentally normal but susceptible to spontaneous tumours. Nature 1992; 356 : 215-21.

13. Hummler E, Cole TJ, Blendy JA, Ganss A, Aguzzi A, Schmid W, Beermann F, Schūtz G. Targeted mutation of the CREB gene: compensation within the CREB/ATF family of transcription factors. Proc Nall Acad Sci USA 1994 ; 91 : 5647-51.
14. Gu H, Marth JD, Orban PC, Mossmann $\mathrm{H}$, Rajewsky K. Deletion of a DNA polymerase $\beta$ segment in $T$ cells using cell type-specific gene targeting. Science $1994 ; 265$ : 1036.

15. Peschon IJ, Behringer RR, Brinster RL, Palmiter RD. Spermatid-specific expression of protamine 1 in transgenic mice. Proc Natl Acad Sci USA 1987; 84 : 5316-9.

16. Murray OR, McCarrey JR, Simon MI. Transcriptionnal regulatory regions of testis-specific PGK2 defined in transgenic mice. Proc Nall Acad Sci USA 1989 ; 86 : 8437 41 .

17. Ewulonu UK, Buratynski TJ, Schimenti JC. Functional and molecular characterization of the transcriptionnal regulatory region of Tcp-10b', a testes-expressed gene from the $t$ complex responder locus. Develop ment $1993 ; 117: 89-95$

18. Behringer RR Crotty DA, Tennyson VM, Brinster RL, Palmiter RD, Wolgemuth DJ. Sequences 5' of the homeobox of the Hox-1.4 gene direct tissue-specific expression of lac $Z$ during mouse development. $D e$ velopment 1993 ; 117 : 823-33.

19. Zambrowicz BP, Zimmermann JW, Harendza J, Simpson EM, Page DC, Brinster RL, Palmiter RD. Expression of a mouse $z f y$ $1 /$ lac $Z$ transgene in the somatic cells of the embryonic gonad and germ cells in the adult testis. Development $1994 ; 120$ : 1549-59.

20. Boissonneault G, Lau YFC. A testis-specific gene encoding a nuclear high-mobilitygroup box protein located in elongated spermatids. Mol Cell Biol 1993 ; 13 : 4323-30.

21. Braun RE, Peschon IJ, Behringer RR, Brinster RL, Palmiter RD. Protamine 3'-untranslated sequences regulate temporal translational control and subcellular localization of growth hormone in transgenic mice. Genes Dev 1989 ; 3 : 793-802.

22. Tamura T, Makino Y, Muramatsu M. Demonstration of a testis-specific trans-acting factor Tet-1 in vitro that binds to the promoter of the mouse protamine-1 gene. J Biol Chem 1992 ; 267 : 4327-32

23. Zambrowicz BP, Palmiter RD. Testis-specific and ubiquitous proteins bind to functionally important regions of the mouse protamine-1 promoter. Biol Reprod 1994 . $50=65-72$.

24. Howard T, Balogh R, Overbeek P, Bernstein K. Sperm-specific expression of angiotensin-converting enzyme (ACE) is mediated by a 91 base-pair promoter containing a CRE-like element. Mol Cell Biol 1993 ; 13 : 18-27.

25. Selten G, Cuypers HT, Boelens W, Robanus-Maandag E, Verbeek J, Domen J, van Beveren C, Berns A. The primary structure of the putative oncogene pim-l shows extensive homology with protein kinases. Cell 1986 ; $46: 603-11$. 
26. Kwon YK, Hecht NB. Binding of a phosphoprotein to the 3' untranslated region of the mouse protamine 2 mRNA temporally represses its translation. Mol Cell Biol 1993 ; $13: 6547-57$.

27. Bellvé AR. Purification, culture, and fractionation of spermatogenic cells. In: Wassarman PW, DePamphilis ML, eds. $M e$ thods in enzymology. New York: Academic Press, 1993 ; 225 : 84-113.

28. Kahn A, Briand P. Établissement de lignées cellulaires par oncogenèse ciblée. $m e ́$ decine/sciences 1991 ; 7 : IV-IX

29. Hoffman MC, Narisawa S, Hess RA, Millan JL. Immortalization of germ cells and somatic testicular cells using the SV40 large T antigen. Exp Cell Res 1992; 201 : 417-35.

30. Cooker LA, Brooke CD, Kumari M, Hofmann MC, Millan JL, Goldberg E. Genomic structure and promoter activity of the human testis lactate dehydrogenase gene. Biol Reprod 1993 ; 48 : 1309-19.

31. Hoffmann MC, Hess RA, Goldberg E, Millàn JK. Immortalized germ cells undergo meiosis in vitro. Proc Natl Acad Sci USA 1994. $91: 5533-7$

32. Peschon IJ, Behringer RR, Cate RL, Harwood KA, Idzerda RL Brinster RL, Palmiter RD. Directed expression of an oncogene to Sertoli cells in transgenic mice using Mullerian inhibiting substance regulatory sequences. Mol Endocrinol 1992 ; 6 : 1403-11.

33. Paquis-Flucklinger V, Michiels JF, Vidal F, Alquier C, Pointis G, Bourdon V, Cuzin F, Rassoulzadegan M. Expression in transgenic mice of the Large $T$ antigen of polyoma virus induces Sertoli cell tumours and allows the establishment of differentiated cell lines. Oncogene $1993 ; 8$ : 2087-94.

34. Rassoulzadegan M, Paquis-Flucklinger V, Bertino B, Sage J, Jasin M, Miyagawa K, van Heyningen $K$, Besmer $P$, Cuzin $F$. Transmeiotic differentiation of male germ cells in culture. Cell $1993 ; 75: 997-1006$.

35. Weintraub $\mathrm{H}$. The MyoD family and myogenesis: redundancy, networks, and thresholds. Cell $1993 ; 75: 1241-4$.

36. Chapman DL, Wolgemuth DJ. Regulation of M-phase promoting factor activity during development of mouse male germ cells. Dev Biol $1994 ; 165$ : 500-6.

37. Iwaoki Y, Matsuda H, Mutter GL, Watrin F, Wolgemuth DJ. Differential expression of the proto-oncogenes $c-a b l$ and $c-m o s$ in developping mouse germ cells. Exp Cell Res $1993 ; 206: 212-9$.

\section{TIRÉS À PART}

F. Cuzin. 